\title{
Kinetic analysis of the temperature dependence of PbSe colloidal quantum dot photoluminescence: Effects of synthesis process and oxygen exposure
}

\author{
Charles A. Foell, ${ }^{1}$ Keith A. Abel, ${ }^{2}$ Frank C. J. M. van Veggel, ${ }^{2}$ and Jeff F. Young ${ }^{1, *}$ \\ ${ }^{1}$ Department of Physics and Astronomy, University of British Columbia, Vancouver, British Columbia, Canada V6T 1 Z1 \\ ${ }^{2}$ Department of Chemistry, University of Victoria, Victoria, British Columbia, Canada V8W 3V6 \\ (Received 29 May 2012; revised manuscript received 23 December 2013; published 29 January 2014)
}

\begin{abstract}
A kinetic model is derived and used to analyze recently published works and new data on the temperature dependence of the spectrally integrated photoluminescence (PL) from thick-film formulations of PbSe colloidal quantum dots (QDs), with particular attention to the effects of air exposure. The model assumes that the excitons thermalize within a ground-state manifold of states and treats the distribution of radiative and nonradiative decay rates within the distribution as generally as possible, while using a minimal number of free parameters. By adjusting the parameters of the model, good fits are obtained for the wide range of integrated PL behaviors reported in [J. Phys. Chem. Lett. 2, 889 (2011); ACS Nano 6, 5498 (2012); Phys. Rev. B 82, 165435 (2010)] and the new data presented in this manuscript. By comparing the extracted parameters we deduce the following: (i) All of the samples in the first two references emit from two distinct clusters of states separated by an energy of 55 to $80 \mathrm{meV}$ regardless of air exposure, while there is only one cluster of emissive states that contributes to the emission reported in the third reference. (ii) In the absence of intentional air exposure, the nonradiative decay from all samples can be described by a single Arrhenius-like process. (iii) Although air-exposure effects are reversible in some samples and irreversible in others, the changes in integrated PL behavior brought about by air-exposure forces the introduction of a common, low-activation-energy nonradiative pathway in all cases. (iv) The low-lying emissive cluster of the two-emissive-cluster samples exhibits behavior similar to the single emissive cluster of the other samples. (v) Many hours of air exposure do not trend either the radiative or nonradiative behavior of the dual-emissive-cluster samples towards the behavior of the single-emissive-cluster samples.
\end{abstract}

DOI: 10.1103/PhysRevB.89.045139

PACS number(s): 78.67.Bf, 73.21.La, 78.55.Qr, 81.07.Ta

\section{INTRODUCTION}

The near-infrared photoluminescence (PL) from PbSe and $\mathrm{PbSe} / \mathrm{CdSe}$ colloidal quantum dots (QDs) with diameters on order 4-6 nm has been studied extensively in solution [1-5] but less so out of solution, where significant applications also exist [6-11]. One obstacle that has hindered progress in gaining a quantitative microscopic understanding of the optical emission from this material system has been the lack of consistency of the experimental results reported by different groups. Chappell et al. [10] and Hughes et al. [11] recently reported temperature-dependent emission spectra from $\approx 5$-nm-diameter oleate-capped $\mathrm{PbSe}$ QDs with apparent contributions from two distinct radiative transitions, the integrated strength of which varies nonmonotonically from 13 to $325 \mathrm{~K}$. Their synthesis is performed in an airfree environment and, depending on procedural details, the temperature dependence of their samples' emission can be irreversibly sensitive, in drop-cast form, to even minute exposures to air.

In contrast, our previously reported temperature-dependent $\mathrm{PL}$ studies [8] using $\approx 4$-nm-diameter $\mathrm{PbSe}$ QDs produced in a similar manner $[9,12,13]$, but with less control over the exposure of the samples to air during synthesis, exhibit monotonic, pure Arrhenius-like decay of integrated PL from 5 to $295 \mathrm{~K}$, and detailed lineshape fits of spectra obtained from several batches over the entire temperature range of 5 to $295 \mathrm{~K}$ were consistent with only a single radiative transition.

\footnotetext{
*young@phas.ubc.ca
}

Previously we independently reported that the PL from these samples degraded at room temperature under exposure to oxygen [13], but that this effect was entirely reversible.

These contrasting results motivated us to carry out further experiments on the full temperature dependence of our samples after exposure to air and to develop a kinetic model that might provide insight into to the similarity and differences between these apparently disparate but nominally very similar $\mathrm{PbSe}$ QD formulations.

\section{SUMMARY OF PHOTOLUMINESCENCE RESULTS}

Table I describes a number of PbSe QD samples and relevant treatments reported in four separate publications, along with a different sample used for new oxygen-exposure studies reported below. Figure 1 contains plots of the temperature dependence of the integrated PL strength corresponding to all of the samples identified in Table I.

The integrated QD PL reported by Chappell et al. and Hughes et al., reproduced in Fig. $1, A_{1}-B_{5}$, is marked by either strong nonmonotonicity and/or explicit increases with increasing temperature. This is in spite of nominally similar QD syntheses for samples $A_{1}$ and $B_{1}-B_{5}$ and no air exposure for samples $A_{1}, A_{2}$, and $B_{1}$. Nonmonotonic PL yield persists even after their samples are exposed to a controlled oxygen environment from minutes to hours. This behavior is attributed to PL contributions from two distinct radiative states at different energies in these QD samples, consistent with spectral lineshape analysis, at least in dilute glass-encased samples, 
TABLE I. Sample descriptions.

\begin{tabular}{lr}
\hline \hline Key & Description (air-exposure extent and originating publication) \\
\hline$A_{1}$ & No air exposure during QD synthesis or as drop-cast, oleic ligands, Hughes et al. [11]. \\
$A_{2}$ & No air exposure during QD synthesis or as drop-cast, alkyl ligands; Hughes et al. [11]. Distinct drop cast from $A_{1}$. \\
$B_{1}$ & No air exposure during QD synthesis or as drop cast; Chappell et al. [10]. Same QD drop-cast sample as traces $B_{2}$ through $B_{5}$. \\
$B_{2}$ & 1 minute of air exposure as drop cast, none during QD synthesis; Chappell et al. [10]. \\
$B_{3}$ & Thirty minutes of air as drop cast, none during QD synthesis; Chappell et al. [10]. \\
$B_{4}$ & Two hours of air exposure as drop cast, none during QD synthesis; Chappell et al. [10]. \\
$B_{5}$ & Twelve hours of exposure as drop cast, none during QD synthesis; Chappell et al. [10]. \\
$C_{1}$ & No air exposure as drop cast, some during QD synthesis; Qiao et al. [8]. \\
$C_{2}$ & No air exposure as drop cast, some during QD synthesis; Qiao et al. [8]. Distinct drop cast from $C_{1}$. \\
$D_{1}$ & No air exposure as drop cast, some during QD synthesis. Same QD drop-cast sample as traces $D_{2}$ and $D_{3}$ (new data). \\
$D_{2}$ & Thirty minutes exposure as drop cast, some during QD synthesis, measured after 2 hours in vacuum (new data). \\
$D_{3}$ & Two hours exposure as drop cast, some during QD synthesis, measured after 48 hours in vacuum (new data). \\
\hline \hline
\end{tabular}

that reveals two peaks separated by several tens of meV, over a range of temperatures.
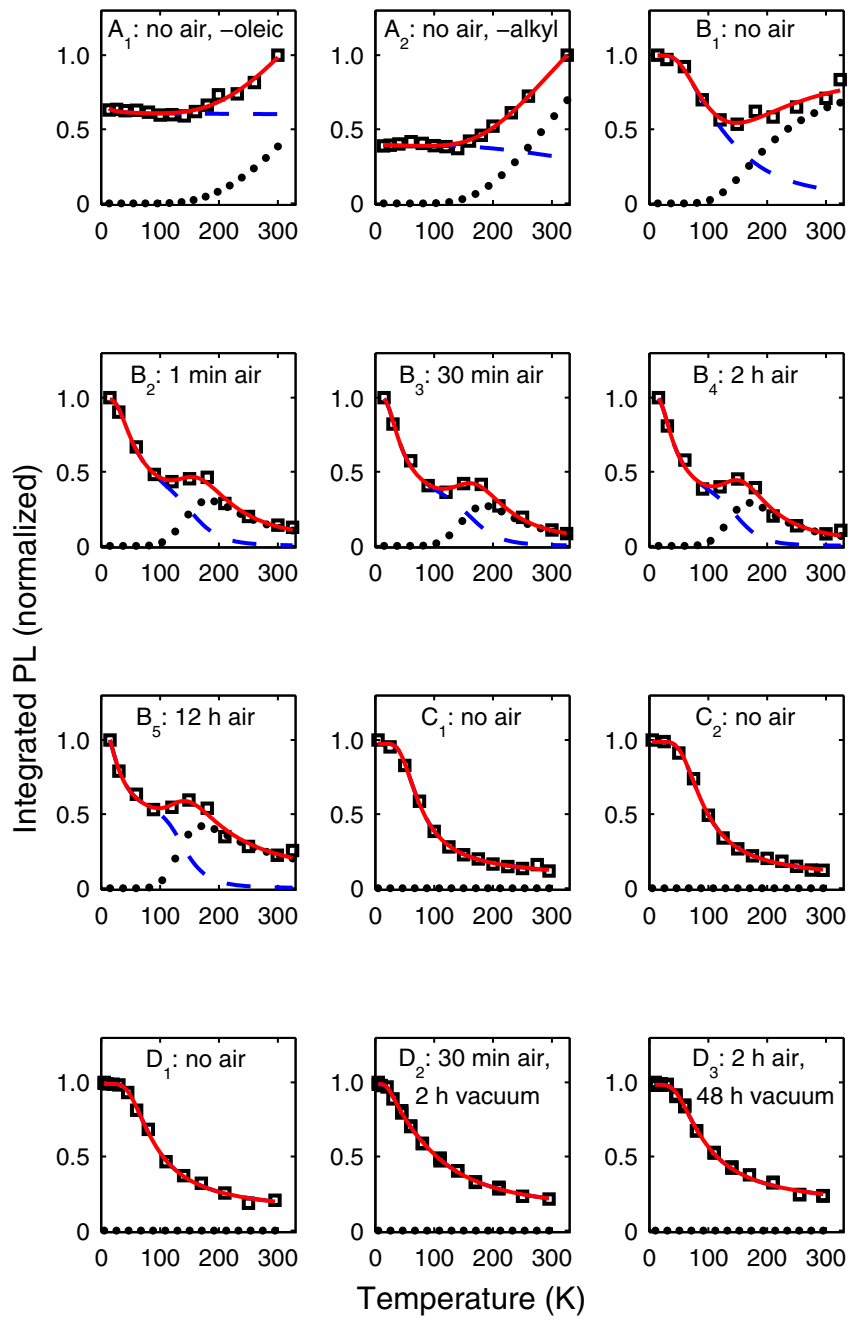

FIG. 1. (Color online) Integrated PL data sets (black squares), normalized to their maximum values, along with best-fit model contributions (calculated below) in solid red and corresponding model PL contributions from each of two possible emissive states or clusters of emissive states in dotted and dashed lines, respectively. Table I contains descriptions of the samples.
Independent models [8,10,11] have been developed to quantify various aspects of the published PL behavior for subsets of the samples listed in Table I. However, we are unaware of any account of a systematic attempt to fit the entirety of this disparate $\mathrm{PbSe}$ nanocrystal temperature-dependent integrated PL data from 5 to $325 \mathrm{~K}$ using a single, physically based model.

To inform quantitative comparisons of samples, we describe below a physically based kinetic model that captures the essential behavior of integrated PL data from all samples listed in Table I. The number of free parameters is minimized, and our analysis of the data based on this model highlights the importance of taking into account parameter covariance when extracting reliable fit parameters consistent with the data.

\section{MODEL}

Various band-structure calculations suggest that the 64-fold degenerate excitonic ground state of bulk $\mathrm{PbSe}$ is spread into a manifold of $S$-S excitonic states in small (3-5 nm diameter) QDs [14]. Intervalley coupling splits the states over an energy range of tens of meV, while electron-hole exchange splitting further lifts the degeneracies by a smaller amount, on order of a few meV [14]. Of these 64 states, only a few have significant oscillator strength.

Assuming that the excitons thermalize to a Fermi-Dirac distribution within the ground-state exciton manifold at all temperatures, the steady-state, spectrally integrated PL emission rate can be expressed as

$$
g_{r}=\int_{\epsilon_{\min }}^{\epsilon_{\max }} \frac{\gamma_{r}(\epsilon) f(\epsilon)}{e^{(\epsilon-\mu) / k T}+1} d \epsilon,
$$

where $f(\epsilon)$ is a sum of $64 \delta$ functions defining the energies of the exciton states within the ground state exciton manifold, spanned by $\epsilon_{\min }$ and $\epsilon_{\max }, \gamma_{r}(\epsilon)$ is the radiative decay rate distribution of the states within the manifold, and the remaining part of the integrand is the Fermi-Dirac occupation factor. Assuming weak (linear), nonresonant optical excitation into the high-lying quasicontinuum of the QD absorption spectrum and rapid decay to the ground-state manifold at a rate $g_{e x}$, the steady-state quasichemical potential $\mu$ of the excitons is 


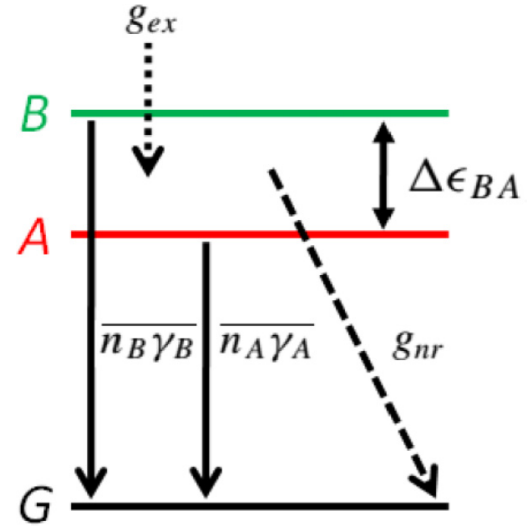

FIG. 2. (Color online) Modeled energy-level arrangement. Levels $A$ and $B$ are in thermal quasi-equilibrium and populated by pumping of higher-lying levels at a rate $g_{e x}$. They consist of $N_{A}$ and $N_{B}$ states, and the averaged net radiative decay rates from the group of $A$ and $B$ states are $\overline{n_{A} \gamma_{A}}$ and $\overline{n_{B} \gamma_{B}}$ respectively. The nonradiative decay rate $g_{n r}$, the nature of which is described in detail in the text, is not specific to levels $A$ or $B$ for the purposes of this kinetic modeling, and is indicated by the dashed arrow.

determined by solving

$$
g_{e x}=\int_{\epsilon_{\min }}^{\epsilon_{\max }} \frac{\left[\gamma_{r}(\epsilon)+\Gamma_{n r}(\epsilon, T)\right] f(\epsilon)}{e^{(\epsilon-\mu) / k T}+1} d \epsilon
$$

for $\mu$ at each $T$, assuming the other parameters are fixed. Here $\Gamma_{n r}(\epsilon, T)$ is the distribution of nonradiative recombination rates for different exciton states in the ground-state manifold, which can be temperature dependent.

In order to limit the free parameters in the kinetic model, we make two assumptions: (i) there are at most two states, or more generally two clusters of closely spaced states, that have significant oscillator strengths, and (ii) the net nonradiative decay rate from the entire manifold can be fit using a temperature-dependent function that does not include any level-specific parameters.

These assumptions are schematically summarized in the energy level diagram of Fig. 2. Nonradiative decay in our model is generally from the collective manifold of excitonic states instead of pathways specific to states within the manifold. Assumption (i) is chosen to be consistent with up-to-date observations of thick film PbSe PL, but can be relaxed if future works reveal otherwise. Assumption (ii) does not preclude a more state-specific interpretation of whatever temperature-dependent function is fit to the nonradiative decay rate. The radiative emission rate is thus approximated:

$$
\begin{aligned}
g_{r} & =\frac{N_{A} \overline{\gamma_{A}}}{e^{\left(\overline{\epsilon_{A}}-\mu\right) / k T}+1}+\frac{N_{B} \overline{\gamma_{B}}}{e^{\left(\overline{\epsilon_{B}}-\mu\right) / k T}+1} \\
& =\overline{n_{A} \gamma_{A}}+\overline{n_{B} \gamma_{B}},
\end{aligned}
$$

where the overline denotes the net or average value of the corresponding quantity associated with the $A$ or $B$ cluster of emissive states.

With reference to Fig. 2, the two clusters of emissive states, $A$ and $B$, have radiative decay rates $\left\{\gamma_{A}\right\}$ and $\left\{\gamma_{B}\right\}$, respectively, and the average or centroid energy of the $B$-state cluster, $\overline{\epsilon_{B}}$, lies $\Delta \epsilon_{B A}$ above the average or centroid energy $\overline{\epsilon_{A}}$ of the $A$ state.

The nonradiative contribution in Eq. (2), denoted by $g_{n r}$, is

$$
g_{n r}=\int_{\epsilon_{\min }}^{\epsilon_{\max }} \frac{\Gamma_{n r}(\epsilon, T) f(\epsilon)}{e^{(\epsilon-\mu) / k T}+1} d \epsilon .
$$

Thus the net PL yield $Y_{\mathrm{PL}}$, which is also proportional to the observed PL, is of the form

$$
\begin{aligned}
Y_{\mathrm{PL}} & =g_{r} / g_{e x} \\
& =\frac{1+\frac{N_{B} \overline{\gamma_{B}}}{N_{A} \overline{\gamma_{A}}} e^{-\Delta \epsilon_{B A} / k T}}{1+\frac{N_{B} \overline{\gamma_{B}}}{N_{A} \overline{\gamma_{A}}} e^{-\Delta \epsilon_{B A} / k T}+\frac{g_{n r}}{N_{A} \overline{\gamma_{A}} e^{-\left(\overline{\epsilon_{A}}-\mu\right) / k T}}},
\end{aligned}
$$

where we have taken the Maxwell-Boltzmann limit of the Fermi-Dirac distribution function. The model was solved more generally, but the experimental excitation conditions and model results are all consistent with this Maxwell-Boltzmann limit.

Using Eq. (5) in the Maxwell-Boltzmann limit, the last term in the denominator of Eq. (7) can be reexpressed as a normalized net nonradiative decay rate,

$$
\begin{aligned}
g_{n r}^{\prime} & =\frac{g_{n r}}{N_{A} \overline{\gamma_{A}} e^{-\left(\overline{\epsilon_{A}}-\mu\right) / k T}} \\
& =\frac{1}{N_{A} \overline{\gamma_{A}}} \int_{\epsilon_{\min }}^{\epsilon_{\max }} \Gamma_{n r}(\epsilon, T) f(\epsilon) e^{-\left(\epsilon-\overline{\epsilon_{A}}\right) / k T} d \epsilon .
\end{aligned}
$$

Excellent fits of the functional form $\eta Y_{\mathrm{PL}}$, for temperatureindependent constant $\eta$, to all data sets were found for $g_{n r}^{\prime}$ set equal to the sum of $N$ Arrhenius terms, i.e.,

$$
g_{n r}^{\prime}=\sum_{j=1}^{N} \Gamma_{j}^{\prime} e^{-\Delta E_{j} / k T},
$$

for $N, \Delta E_{j}$, and $\Gamma_{j}^{\prime}$ dependent upon sample type, but in all cases only one or two nonradiative pathways are needed.

By modeling the manifold's net nonradiative decay using Eq. (10), the only influential state energy for the purposes of our fitting methodology is that of the $B$-state cluster, relative to that of the $A$-state cluster, i.e., $\Delta \epsilon_{B A}$; energies $\overline{\epsilon_{A}}$ and $\epsilon_{\min }$ become irrelevant. Individual terms in Eq. (10) have no specific association with the exciton states in the manifold: any one term could in principle represent some combination of a thermal occupancy factor for a particular state (bright or dark) and an associated nonradiative decay rate, with the implication that there are $N$ clusters of states through which most of the nonradiative recombination occurs. However, there are no such attributions implied by this more general functional fit.

\section{FIT METHODS AND EXTRACTED PARAMETERS}

Extracted parameter values are plotted in Fig. 3. In those plots, a vertical bar for a particular parameter and particular sample represents the range of that parameter value for which there exists a complimentary set of parameters such that the model fit satisfies $\chi^{2}-\min \left(\chi^{2}\right) \leqslant 4$, where $\chi^{2}=$ $\Sigma$ (observed - model $)^{2} / \sigma^{2}$, "observed" is the observed PL, "model" is the model PL in the Maxwell-Boltzmann limit $\left[\eta Y_{\mathrm{PL}}\right.$ for $Y_{\mathrm{PL}}$ of Eq. (7)], $\sigma=0.02 \max \left(I_{\mathrm{PL}}\right)$, and $\min \left(\chi^{2}\right)$ is evaluated at the global best fit, obtained according to 

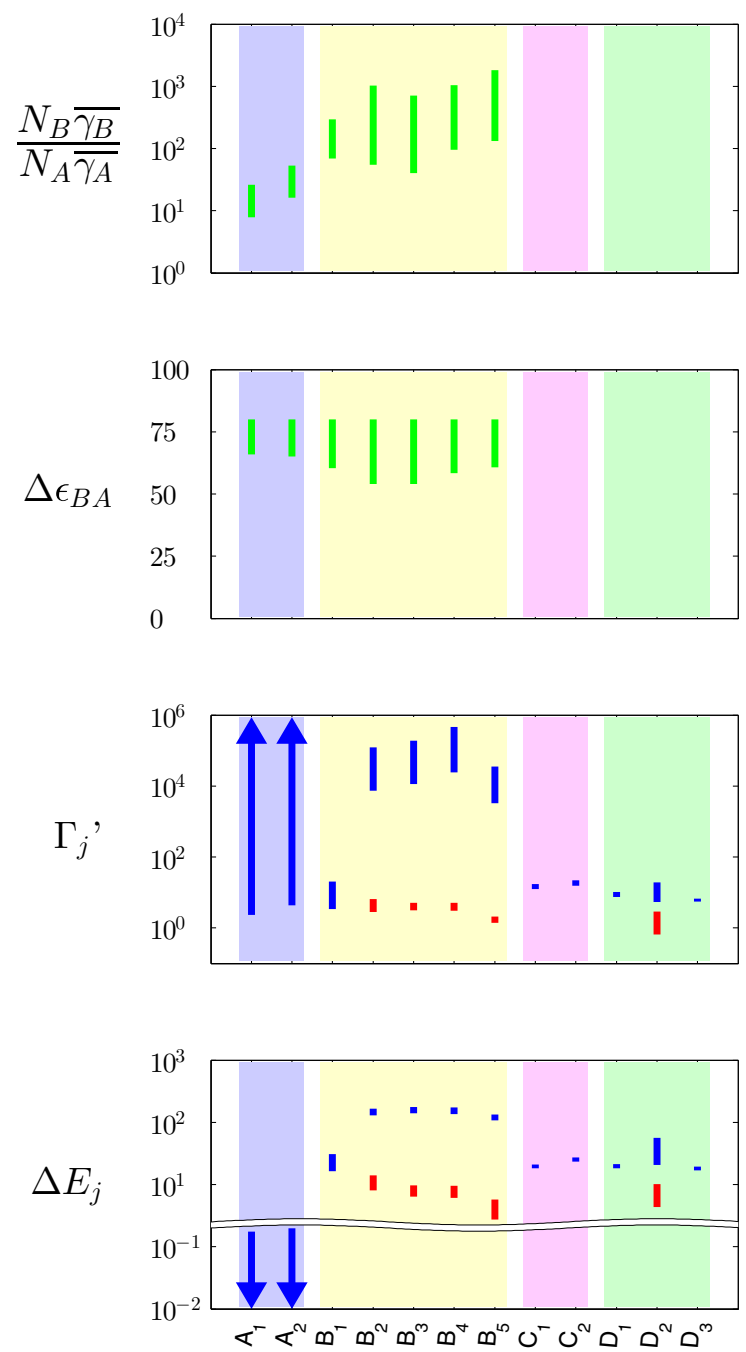

FIG. 3. (Color online) Bars represent the ranges of parameter values that, upon substitution into Eq. (7), using Eq. (5), generate or nearly generate the model curves in Fig. 1. Rates are normalized as described in the text, and energies are in meV. Sample labeling is as in Fig. 1 and Table I. Parameter $\Delta \epsilon_{B A}$ is restricted from above to $80 \mathrm{meV}$ for reasons described in the text. Rates sufficiently small to render their corresponding energies meaningless are omitted, along with those energies.

the procedures described below. The number of nonradiative pathways used to fit to any particular curve was chosen such that $\min \left(\chi^{2}\right)$, normalized to the number of data points for that curve, was less than three. This threshold was found to admit best fits that captured qualitative behavior of all the sample curves, especially relating to the effects of air exposure.

The sole constraint imposed upon the parameters is an upper limit of $80 \mathrm{meV}$ for $\Delta \epsilon_{B A}$, based on the spectral range over which the PL was integrated and allowing for possible differences in the Stokes shifts associated with the two radiative levels in sample series $A$ and $B$. Otherwise, the parameter search domains correspond to the plotting ranges in Fig. 3.

The first segment of our best fit algorithm involves (1) sampling a set of parameters logarithmically from the param- eter search domain, (2) applying the Levenberg-Marquardt algorithm with this set of parameters as an initial condition to determine the local best-fit parameters, (3) repeating (1) and (2) a total of $10^{3}$ times, and (4) associating the best fit of these local best fits with the global best fit. This global best fit was corroborated via application of local minimization to a parameter search domain grid (the latter contrasted with random sampling of the parameter search domain). The logarithm of the likelihood function was found to be quadratic in most parameters near the best-fit points.

Red lines in Fig. 1 are the model curves corresponding to the best-fit parameters found by this procedure. Dotted and dashed lines are plots of the individual model $A$-state and $B$-state contributions from each state for the same best-fit parameters.

The second segment of our fit routine-generation of the vertical parameter bars in Fig. 3-consisted of (5) dividing up each parameter search domain into a set of many narrow subdomains, (6) restricting, parameter by parameter of a particular sample, that parameter value to one of those narrow subdomains, (7) sampling a complimentary set of parameters logarithmically from the large parameter search domain, (8) applying the Levenberg-Marquardt algorithm to determine a local best fit, (9) repeating (7) and (8) at total of $10^{2}$ times, (10) identifying the best fit of those local best fits, (11) checking to see if $\chi^{2}-\min \left(\chi^{2}\right) \leqslant 4$ is satisfied for the best of those local best fits, and (12) repeating steps (6) through (12) for every other narrow subdomain. The vertical bars in Fig. 3 are lines that extend from the minimum to maximum parameter values with which some $\chi^{2}-\min \left(\chi^{2}\right) \leqslant 4$ exists, as found in steps (5) through (12).

Figure 1 indicates that good quality fits to the data are obtained using the Maxwell-Boltzmann limit of the model. This limit should apply only when $g_{e x} / N_{A} \overline{\gamma_{A}} \ll 1$, in which case the results are independent of $g_{e x}$. We estimated $g_{e x}$ for both our data sets and those of Chappell et al. using measured laser powers and spot sizes, along with absorption coefficients from the literature [15]. The spontaneous emission rate of the lower emissive state was estimated from lifetime measurements of our drop-cast films and dielectric modeling we previously employed [16]. For both our samples and those of Chappell et al., we estimate $g_{e x} / N_{A} \overline{\gamma_{A}} \ll 10^{-1}$. Furthermore, if $g_{e x}$ is increased to the point where the Maxwell-Boltzmann approximation breaks down, it becomes very difficult to fit any data set (impossible in the strongly degenerate limit).

\section{FIT RESULTS}

With reference to the parameter summary in Fig. 3, the most significant difference between the various samples is that high-quality fits required $N_{B} \overline{\gamma_{B}} / N_{A} \overline{\gamma_{A}} \gg 1$ for all Chappell et al. and Hughes et al. samples, and $N_{B} \overline{\gamma_{B}} / N_{A} \overline{\gamma_{A}} \ll 1$ for all of our samples. This is consistent, in the former case, with the prominent mid-temperature increase in PL and doublepeaked mid-temperature spectra, and in the latter case, with our observation of only a single emission peak within the few-meV resolution of previously reported spectra [8]. Also, although a trend in $N_{B} \overline{\gamma_{B}} / N_{A} \overline{\gamma_{A}}$ with oxygen exposure is admissible, any such trend does not follow necessarily from our kinetic 
analysis, and it is further clear that increasing oxygen exposure does not trend $N_{B} \overline{\gamma_{B}} / N_{A} \overline{\gamma_{A}}$ in their samples towards the value for our samples.

Fits require the $A-B$ energy separation for series $A$ and $B$ samples to be $\Delta \epsilon_{B A} \approx 55$ to $80 \mathrm{meV}$. It should be noted that the $\Delta \epsilon_{B A}$ value obtained from fits to the no-exposure data agrees reasonably well with the separation of the emission lines in the solution spectra, but not in the drop-cast samples. This is to be expected since thermalization of the exciton populations is known [8] to have a strong influence on the temperaturedependent Stokes shift of the peak PL emission energy, while there should be minimal effects of thermalization on the spectra obtained in a dilute solution, where the Förster interaction strength is negligible.

For all samples that were not intentionally exposed to oxygen, the integrated PL curves can be fit assuming only a single nonradiative pathway. The associated parameters of this nonradiative pathway (an activation energy around a few tens of meV and normalized prefactor on the order of several to a few tens) are quite similar for the unexposed sample from Chappell et al. $\left(B_{1}\right)$ and our unexposed samples $(C$ series and $D$ series excluding $D_{2}$ ). Nonradiative decay in series $A$ samples is consistent with a single nonradiative pathway with a considerably lower activation energy. Without information regarding the absolute quantum yield in the case of series $A$ samples, it is not possible from the fitting procedure to separate small differences in assumed activation energy and the corresponding prefactor for this single nonradiative decay process (hence the unbounded arrows for series $A$ samples). Introduction of a second sub-10-meV nonradiative pathway increases fit quality for the Chappell et al. unexposed sample $\left(B_{1}\right)$, but the difference in fit quality is small. The slight increase in integrated PL at low temperatures in the $A_{2}$ sample is not captured with our model, but a third, relatively dark level, just slightly below the $A$ level in energy, could be introduced to capture this behavior.

Samples intentionally exposed to air $\left(B_{2}-B_{5}\right.$ and $\left.D_{2}\right)$ absolutely require the inclusion of two nonradiative pathways for adequate fits. One of these new pathways has a low (sub10-meV) activation energy, with a corresponding prefactor in the range of 1 to 10, indicated by the red bars in Fig. 3. In our air-exposed sample $\left(D_{2}\right)$, the parameters associated with the second nonradiative pathway are not dramatically different than before air exposure, whereas in the case of samples $B_{2}-B_{5}$, the second pathway has a very high activation energy ( $>100 \mathrm{meV}$ ), and high normalized prefactor (100s of thousands). There is no indication of the need for a high-energy pathway in any of the unexposed or our air-exposed samples. We note that while the low-energy nonradiative pathway is common amongst all air-exposed samples, Chappell et al. report irreversibility of this air-exposure effect for their samples, in stark contrast to the reversibility of the impact of our air exposure (see sample $D_{3}$ compared to $D_{1}$ and $D_{2}$ ). Finally, we note that inclusion of a third nonradiative pathway with an activation energy of several tens of meV increases the quality of fit for the $B_{2}-B_{5}$ samples; but again, the improvement is minor.

Regarding phonon-mediation of nonradiative decay, Chappell et al. and Hughes et al. assumed a $N_{p h}$-phonon absorption functional form for their low-energy, nonradiative pathway [10,11], and they reported fits suggested $N_{p h}=$ $4 \pm 2$. In cases where present, if we replace the severalto-ten meV nonradiative Arrhenius pathway $\left(\Gamma^{\prime} e^{-\Delta E / k T}\right)$ with a one-phonon emission functional form, $\Gamma_{p h}^{\prime}[1-$ $\left.\exp \left(-E_{p h} / k T\right)\right]^{-1}$, similar-quality fits to those shown in Fig. 1 can be obtained for a phonon energy of $\approx 5$ to $10 \mathrm{meV}$. From this analysis, there is no preference for the single phonon versus low-activation energy Arrhenius process, but it is nevertheless unambiguous that a nonradiative decay channel with a low $(<10 \mathrm{meV})$ activation energy is required to fit all of their and our oxygen-exposed samples. The lack of preference between these two functional forms can be attributed to substantial parameter covariance.

\section{DISCUSSION}

The behavior of the lower-energy $A$ state for samples from series $B$ is very similar to that of the only radiative state that manifests itself in our samples (series $C$ and $D$ ). With no oxygen exposure, the integrated PL from the $A$ transition in sample $B_{1}$ is almost identical to that observed in all series $C$ and $D$ samples save for the the one exposed to air $\left(D_{2}\right)$. In the case of all oxygen-exposed samples from series $B$ and $D$, the change in behavior of the $A$-state contribution is very similar to the change in behavior of our single emissive state in sample $D_{2}$, up to temperatures above approximately $125 \mathrm{~K}$, beyond which point the $A$-state contribution in oxygen-exposed $B$ series samples decays more rapidly than in sample $D_{2}$. It would appear that oxygen introduces a new, low-activation-energy nonradiative decay path for state $A$, and our single emissive state, which causes more rapid (than in the absence of oxygen exposure) falloff of the emission as temperature, is increased from approximately $5 \mathrm{~K}$. The difference in behavior at higher temperatures for the $A$-state contribution from samples in series $B$ is apparently due to an additional, high-activationenergy nonradiative pathway that oxygen exposure causes in those samples. It seems plausible that this high-activationenergy nonradiative channel is somehow tied to the $B$ state that is apparent in all series $A$ and $B$ samples, but absent in our series $C$ and $D$ samples.

One explanation for the absence of radiative and nonradiative evidence of the $B$ state in our samples is that the next-highest-lying state with significant oscillator strength in our samples has a $\Delta \epsilon_{B A}$ much larger than $\approx 80 \mathrm{meV}$. An alternate explanation for the lack of radiative evidence of the $B$ state in our samples, is the possible impact of nanoparticle shape or symmetry on the oscillator strengths [17] of the states within the ground-state exciton manifold. Positing explanations for these conjectures raises the issue of the nature of both the $A$ and $B$ states; are either or both "intrinsic" excitonic states constructed from the one electron and one hole single-particle states typically calculated for these QDs, or are either or both associated with defects, either bulk like or surface like? Based on most published band-structure calculations for these systems, it is hard to identify an "intrinsic" state that could be associated with the $A$ state in this analysis, since almost all of these calculations conclude that the ground states of the intrinsic manifold are "dark," with the first "bright" states located on the order of 10 to $20 \mathrm{meV}$ above the intrinsic ground state. Since, with the exception of the Hughes et al. 
sample $A_{2}$, and possibly other Hughes et al. samples similar to the $A$ series, there is no experimental evidence of increasing PL emission from temperatures of $5 \mathrm{~K}$, the $A$ state must be close to the energetically lowest available excitonic state in the QDs, whatever its nature. In the case of the Hughes et al. sample $A_{2}$, the weak rise in integrated PL at low temperature does suggest that the $A$ state in those samples lies slightly above the ground state, but not by the amount suggested by most published band-structure calculations. Thus, to the extent that current band-structure calculations accurately capture the intrinsic properties of these QDs, this suggests the $A$ state in series $B$ samples, and the only emissive state in our QDs, is a defect state with non-negligible oscillator strength, or that some detail of the nanoparticle structure influences the theoretical oscillator strengths in such a way that low-energy exchange-split states have large oscillator strengths. If the impact of symmetry is sufficient to impact the low-energy $A$ state, it may not be so surprising that the higher-energy $B$ state's oscillator strength could be quite sensitive to sample synthesis details.

Further experiments and theoretical calculations that include defects and particles of various symmetries are required to explain the nature of the optically active states in $\mathrm{PbSe}$ QDs, and the reversibility of the influence of oxygen in some samples, and its irreversible nature in other samples.

\section{CONCLUSION}

A kinetic model that allows for luminescent emission from up to two bright clusters of states, and nonradiative decay from any of the excitonic states in the lowest manifold of PbSe QD thin films can accurately reproduce a diverse range of measured temperature-dependent integrated PL emission data from 5 to $325 \mathrm{~K}$. A thorough statistical analysis of the best-fit parameters (at most six, and in some cases as few as two), including their covariance, reveals the commonalities and differences in the optical emission properties of nominally similar PbSe QD samples grown in different laboratories, including their response to oxygen exposure.

One set of samples (labeled $C$ and $D$ ) effectively emit from only a single cluster of bright states that have energies at or very close to the lowest of the accessible excitonic states. In the absence of intentional oxygen exposure, the nonradiative decay from these samples is well described by a single Arrhenius process with an activation energy on order of $20 \mathrm{meV}$. The other sets of samples (labeled $A$ and $B$ ) exhibit significant emission from primarily two clusters of states separated by $\approx 55$ to $80 \mathrm{meV}$, with the lower-energy state very close to the bottom of the manifold of accessible states. In the absence of intentional oxygen exposure, samples $A$ and $B$ either exhibit the same $20 \mathrm{meV}$ activated nonradiative decay behavior of samples $C$ and $D$, or a nonradiative decay with negligible activation energy, depending on the organic capping layer.

Oxygen exposure of samples $B, C$, and $D$ changes their behavior in a way that can be described by introducing a second, distinct Arrhenius-like nonradiative decay channel with a relatively low activation energy. The biggest difference between samples $B$ and samples $C$ and $D$ in this regard, is that the effects of oxygen exposure on samples $B$ is irreversible, while it is reversible for samples $C$ and $D$.

This analysis and sample comparison suggests that the lower-energy transition in samples $A$ and $B$ shares many attributes of the single emissive sate in samples $C$ and $D$. The fact that the energy of this bright state is very close to the bottom of the ground manifold of accessible exciton states is difficult to reconcile with existing calculations of the oscillator strength distribution within the "intrinsic" groundstate manifold of PbSe QDs. This, together with the apparently fickle nature of the higher-energy emissive state evident in one set of samples but absent in the other suggests that either (i) the low-energy emissive state is associated with defects, and/or (ii) that current calculations of band structure and oscillator strengths fail to capture important details, perhaps associated with particle morphology, that have yet to be experimentally determined.

Further experimental specification of the precise physical makeup of these small QDs, and further studies of the impact of oxygen exposure are required to better understand these PbSe QDs.

\section{ACKNOWLEDGMENTS}

We gratefully acknowledge the insight regarding bandstructure models in this system provided by Pawel Hawrylak and Oleksandr Voznyy. We also acknowledge the financial support of the Natural Sciences and Engineering Research Council and the Canadian Institute for Advanced Research.
[1] H. Du, C. Chen, R. Krishnan, T. D. Krauss, J. M. Harbold, F. W. Wise, M. G. Thomas, and J. Silcox, Nano Lett. 2, 1321 (2002).

[2] B. L. Wehrenberg, C. Wang, and P. Guyot-Sionnest, J. Phys. Chem. B 106, 10634 (2002).

[3] J. M. Pietryga, D. J. Werder, D. J. Williams, J. L. Casson, R. D. Schaller, V. I. Klimov, and J. A. Hollingsworth, J. Am. Chem. Soc. 130, 4879 (2008).

[4] H. Liu and P. Guyot-Sionnest, J. Phys. Chem. C 114, 14860 (2010).

[5] O. E. Semonin, J. C. Johnson, J. M. Luther, A. G. Midgett, A. J. Nozik, and M. C. Beard, J. Phys. Chem. Lett. 1, 2445 (2010).

[6] J. M. Harbold and F. W. Wise, Phys. Rev. B 76, 125304 (2007).
[7] C. G. Christova, J. W. Stouwdam, T. J. Eijkemans, A. Y. Silov, R. W. van der Heijden, M. Kemerink, R. A. J. Janssen, and H. W. M. Salemink, Appl. Phys. Lett. 93, 121906 (2008).

[8] H. Qiao, K. A. Abel, F. C. J. M. van Veggel, and J. F. Young, Phys. Rev. B 82, 165435 (2010).

[9] K. A. Abel, H. Qiao, J. F. Young, and F. C. J. M. van Veggel, J. Phys. Chem. Lett. 1, 2334 (2010).

[10] H. E. Chappell, B. K. Hughes, M. C. Beard, A. J. Nozik, and J. C. Johnson, J. Phys. Chem. Lett. 2, 889 (2011).

[11] B. K. Hughes, D. A. Ruddy, J. L. Blackburn, D. K. Smith, M. R. Bergren, A. J. Nozik, J. C. Johnson, and M. C. Beard, ACS Nano 6, 5498 (2012). 
[12] C. B. Murray, S. Sun, W. Gaschler, H. Doyle, T. A. Betley, and C. R. Kagan, IBM J. Res. Dev. 45, 47 (2001).

[13] J. W. Stouwdam, J. Shan, F. C. J. M. van Veggel, A. G. Pattantyus-Abraham, J. F. Young, and M. Raudsepp, J. Phys. Chem. C 111, 1086 (2007).

[14] J. M. An, A. Franceschetti, and A. Zunger, Nano Lett. 7, 2129 (2007).
[15] I. Moreels, K. Lambert, D. De Muynck, F. Vanhaecke, D. Poelman, J. C. Martins, G. Allan, and Z. Hens, Chem. Mater. 19, 6101 (2007).

[16] C. A. Foell, E. Schelew, H. Qiao, K. A. Abel, S. Hughes, F. C. J. M. van Veggel, and J. F. Young, Opt. Express 20, 10453 (2012).

[17] S. V. Goupalov, Phys. Rev. B 79, 233305 (2009). 